\section{NSAID bei Rückenschmerzen haben wohl keinen Effekt}

Bei Rückenschmerzen werden gerne nicht steroidale Entzündungshemmer verordnet. Der Einsatz sollte zeitlich limitiert erfolgen - oder vielleicht gleich nach Alternativen gesucht werden. Die Ergebnisse eines Cochrane-Reviews zur Wirksamkeit sind nämlich ernüchternd.

Be ei Schmerzen im unteren Rücken empfehlen Leitlinien den kurzfristigen Einsatz von nicht steroidalen Entzündungshemmern (NSAID). Um die aktuelle Evidenz für diese Empfehlung zu überprüfen, führte die Cochrane Collaboration ein systematisches Review

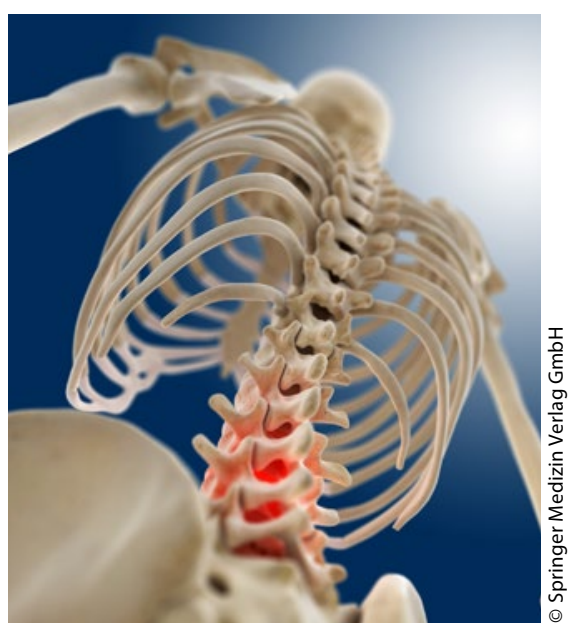

Bei Schmerzen im unteren Rücken sind NSAID wenig erfolgversprechend. mit Metaanalyse durch, das jetzt in der Zeitschrift JAMA kurz zusammengefasst wurde.

Einbezogen in die Metaanalyse waren 13 randomisierte klinische Studien mit insgesamt 4.807 Patienten, die zwischen 1982 und 2013 publiziert worden waren. Beim primären Studienziel Reduktion der Schmerzintensität ergab sich ein kleiner, statistisch signifikanter Vorteil zugunsten der NSAID, allerdings bei insgesamt niedriger Evidenzqualität. Die durchschnittliche Vorher-NachherDifferenz der Schmerzintensität betrug -6,97 (95\%-Konfidenzintervall [KI] $-10,74$ bis $-3,19)$, gemessen mit einer von 0 bis 100 skalierten visuellen Analogskala. Die mediane Nachbeobachtungszeit betrug 56 Tage mit einem Interquartilsabstand von 13-91 Tagen.

In vier Studien wurden zusätzlich Verbesserungen bei krankheitsbedingten Beeinträchtigungen ermittelt. Auch hier ergab sich bei insgesamt niedriger Evidenzqualität ein etwas größerer Nutzen für die NSAID mit einer durchschnittlichen Differenz zu Therapieende im Ver- gleich zum Behandlungsbeginn von $-0,85$ (95\%-KI -1,30-0,40), gemessen mit einer 25-Punkte umfassenden visuellen Analogskala.

Bei beiden Wirksamkeitsparametern waren die errechneten Unterschiede allerdings geringer als die minimale Differenz für die klinische Relevanz. Nach Ausschluss von Studien mit hohem BiasRisiko verlor sich zudem die Signifikanz der Differenzen sowohl bei der Schmerzintensität als auch bei der Beeinträchtigung. Bei den Nebenwirkungen zeigte sich kein Verum-Placebo-Unterschied.

In einer randomisierten Vergleichsstudie war Celecoxib gegenüber Tramadol überlegen. In einer weiteren Vergleichsstudie reduzierte ein zuhause durchgeführtes Bewegungsprogramm die körperlichen Beeinträchtigungen besser als NSAID, Unterschiede bei der Schmerzintensität waren in dieser Studie nicht zu erkennen.

Fazit: Insgesamt ist der analgetische Effekte von NSAID bei Patienten mit Rückenschmerzen gering - in einer Metaanalyse von randomisierten Studien ergab sich ein minimaler, klinisch aber nicht relevanter Effekt. Nun sollte nach Parametern gesucht werden, mit denen sich potenzielle NSAID-Responder besser identifizieren lassen.

Dr. Barbara Kreutzkamp

Enthoven WTM et al. NSAIDs for chronic low back pain. JAMA 2017; 317:2327-8

\section{Neu aufgetretene persistierende Kopfschmerzen: Psychiatrische Komorbidität überdurchschnittlich}

\author{
Neu aufgetretene persistierende Kopfschmerzen betreffen möglicherweise \\ vor allem solche Patienten, die unter psychosozialem Stress leiden, hervor- \\ gerufen zum Beispiel durch Depressionen, Angst oder Katastrophisieren. Eine \\ erste Studie überprüfte das.
}

D er neu aufgetretene persistierende tägliche Kopfschmerz (new daily persistent headache, NDPH) ist ein neuer Diagnose-Subtyp des chronischen täglichen Kopfschmerzes. Der Beginn dieser persistierenden Kopfschmerzen vom Spannungs- oder Migränetyp muss definitionsgemäß vom Patienten eindeutig benannt werden können und bereits mindestens über drei Monate anhalten. Als Ereignisse, die dem abrupten Beginn vorangehen, wurden unter anderem virale Infektionen, Traumata oder Operationen identifiziert. Vermutlich liegen jedoch zusätzliche Faktoren vor, die die Schmerz-Chronifizierung begünstigen. Dazu dürfte auch emotionaler Dysstress gehören, zum Beispiel im Rahmen verschiedener psychiatrischer Erkrankungen. Eine monozentrische Querschnittsstudie ging der Frage nach.

Einbezogen wurden die Daten von 55 konsekutiven Patienten mit einer NDPH-Diagnose mit Kopfschmerzen sowohl vom Spannungs- als auch Migränetyp; das Vorliegen eines Analgetikainduzierten Kopfschmerzes wurde ausgeschlossen. Anhand des Patient Health Questionnaire-9 und der Generalized Anxiety Disorder Scale-7 machten die Patienten Angaben zu Depressions- und 